\title{
HiSTORIA Y POLÍTICA EN ARISTÓTELES: \\ Constitución de Atenas y Política*.
}

\author{
Héctor Edo. García Cataldo \\ Universidad de Chile. Chile
}

Resumen: Esta exposición llama la atención sobre la vinculación estrecha que hay entre historia y política en la filosofía política aristotélica, así como mostrar que Aristóteles, metodológicamente, se sirvió de la investigación histórico-política para levantar su propuesta de una politeía ideal, en la que incorporó a la clase media como elemento indispensable de estabilización, superando la concepción platónica de la guerra y el predominio, en el mundo griego, de las guerras civiles, poniendo como fundamento de la vida política la amistad (la filía).

Palabras claves: Historia, política, politeía, stásis, clase media, amistad

Abstract: This article highlights the close link between history and politics in Aristotelian political philosophy, and shows how Aristotle, as part of his method, used his own investigation on history and politics to uphold his proposal of an ideal politeia, in which to include the middle class as an indispensable factor of stability. He thereby overcame both the Platonic conception of war, and the predominance of civil wars in the Greek world. For Aristotle friendship (filia) was the basis of political life.

Key words: History, politics, politeia, stasis, middle class, friendship.

Recibido: 26.11 .08 - Aceptado: 23.01 .09

Correspondencia: Héctor García Cataldo. Licenciado en Filología Clásica con opción helénica y latina. Magister en Historia con mención en Historia Europea y candidato a doctor en Filosofía hegarcia@uchile.cl Tel. 32-2495459. Profesor Universidad de Chile, Pontificia Universidad Católica de Valparaíso, Universidad Playa Ancha. 


\section{Historia y política}

Dedíamos - y de hecho deberíamos- comenzar primero por
explicar el sentido que tienen las palabras expresadas en el
título. Eso sería demasiado. Todo el mundo ha escuchado y por lo tanto le es familiar, qué es la historia. Ni qué decir de nuestra palabra política. Sin duda que ella es no sólo más permanente, sino también siempre actual y de tanto que se sabe de ella que despierta hasta una cierta repulsión, y no sin razón. Desde que nacemos ya sabemos de ella, porque en ella estamos. Desde lo que es familiar, es decir, desde aquel núcleo inseparable a la polis, el ser y el depender de ella constituyen el fundamento de nuestro accionar en el mundo. Intentar entrar en la explicación de estos simples fenómenos propiamente anthrópicos sería una simple majadería, me conformaría sólo con verlos en el ejercicio, es decir, en vigor en algún momento de la historia.

Más imposible aún explicar esto, en Aristóteles. De aquí la referencia complementaria a la Constitución de Atenas y a la Política.

Respecto de la primera sólo quisiera destacar que es el más reciente descubrimiento en torno a obras del estagirita. En 1891 F. G. Kenyon publicó el famoso papiro de Londres -como se le conoce en la actualidad- el cual de un modo extenso y en buen estado de conservación contenía la Constitución de Atenas ${ }^{1}$. Este hallazgo vino a refrendar un fragmento papiráceo que se encontraba en el Museo de Berlín y que en 1880 había dado a la publicidad el profesor F. Blass ${ }^{2}$. Un año más tarde, en 1881, el profesor Th. Bergk dedica un artículo a este fragmento, atribuyendo la paternidad del mismo a Aristóteles $^{3}$. Como era de esperarse no tardaron en aparecer las tesis contrarias, negando dicha paternidad, y atribuyéndola a lo más a la escuela o a alguno de los discípulos de Aristóteles ${ }^{4}$. En la actualidad está confirmada la paternidad de la obra al filósofo. No debemos perder de vista que esta obra es parte de un conjunto mucho mayor y que se refiere a la compilación de

(*) Este trabajo fue leido en el CONGRESO INTERNACIONAL ARISTÓTELES: FILOSOFÍA PRÁCTICA. Pontificia Universidad Católica de Valparaíso. Chile, ¿......? Se presenta aquí con ligeras modificaciones

${ }^{1}$ Editio princeps de F. G. KENYON, Aristotle on the Constitution of Athens. Londres, 1891.

2 Neue Papyrusfragmente eines Historikers im Aegytischen Museum zu Berlin. Hermes 15 [1880], 366-382.

${ }^{3}$ Zur Aristotelischen Politie der Athener. Rheinisches Museum 36 [1881], 87-115.

${ }^{4}$ Cfr., en esta polémica HIGNETT, A History of the Atenían Constitution: to the end of the fifth century B.C. At the Clarendon Press. Oxford, 1958. 
cuerpos legales de 158 constituciones de las más diversas ciudades helenas y pueblos bárbaros y cuyo conjunto constituía el marco de reflexión para elaborar una teoría política que diera respuesta a la crisis y al resquebrajamiento total en el que había ya desaparecido la polis tradicional. Respecto de la Política, sin embargo, sólo me limito a señalar que allende la discusión detallada en torno al período de su escritura, con toda seguridad Aristóteles seguía precisando estos temas en la última etapa de su vida, o dicho de otro modo, correspondería al período de su segunda estadía en Atenas y comprendería, prácticamente, los diez últimos años de su vida. Y es esto algo que comparten en común ambos trabajos.

A ese espacio acotado al que me refiero, los griegos llamaron pólis y al que en él se movía lo llamaron polítes y a las cosas y acciones que emprendía las llamó simplemente politiká. Desde entonces historia y política han formado una unidad indisoluble, aunque en el ejercicio vulgar nos refiramos a ellas como cosas separadas, de modo que podríamos decir que las tà politiká son el material y el fundamento del que se constituye la historia $\mathrm{y}$, por consiguiente todo teorizar presente sobre la política no puede (no debe) desligarse del percurso histórico de la misma. Aristóteles, más que ninguno en la Antigüedad, tuvo claro este sentido de los asuntos políticos, haciendo de él parte constitutiva de su método investigativo, es decir, partió de un principio general referido al nacimiento y desarrollo histórico del fenómeno llamado polis cuando afirmaba con toda claridad que si alguien mirara los acontecimientos (las cosas), brotando desde su origen, vería con muchísima más claridad en el interior de esos mismos acontecimientos ${ }^{5}$, y este es un principio que se puede referir a todo orden de cosas.

Historia y política en Aristóteles, remiten a un contexto, a un tiempo y a un pensador determinados, más de dos milenios nos separan, de modo que intentar reconstruir en sus detalles aquella historia-política es un tanto iluso. No obstante, ¿es posible oír algo que nos aproxime a aquélla, en tanto que presente? Por ejemplo, encuentro el siguiente texto en el discurso Acerca de la Paz (Perì tes eirénes) de Demóstenes:

Ciertamente veo, atenienses, que los acontecimientos presentes tienen mucha dificultad y perturbación, no sólo por haber perdido muchas cosas y no sacar ninguna utilidad pese a hablar bien de estos hechos,

\footnotetext{
${ }^{5}$ P. 1. 2. 1252 a 24-26. Se trata del "método genético", referido al proceso de desarrollo de las cosas.
} 
sino también acerca de las cosas que nos quedan, no todos consideramos lo útil de una misma manera con respecto a los mismos asuntos, sino que a unos parece de este modo y a otros de otra manera ${ }^{6}$

No pensemos que esta cita tiene por objeto contraponer a ambas figuras, representativas de la Antigüedad helena, aún siendo un recurso metodológico legítimo, no es el propósito. Simplemente queremos hacer sonar unas palabras y entre ellas los acontecimientos presentes (tà parónta prágmata), que Demóstenes caracteriza como de mucha dificultad (dyskolían) y perturbación (tarachén). Se trata de una constatación in situ, en el otoño del año 346/5 a. C. ¿Qué ha pasado en aquel presente de acontecimientos, que es también el presente del que -mutatis mutandis- habla Aristóteles? Los detalles de esta segunda parte del siglo IV son sobradamente conocidos y, por lo mismo, me eximo de entrar en ellos.

Ambas figuras son herederas de los acontecimientos de un tiempo compartido, y el orador sintetiza magistralmente el problema fundamental que encierra todo presente en tanto que acontecimientos, desde el mirar propio del hombre que interviene y juzga ese mismo presente. Demóstenes explicó que la dificultad y perturbación se debían a que Atenas había capitulado ante el poderío macedonio, aceptando y reconociendo que Filipo formara parte del Consejo anfictiónico y porque ya, territorialmente, había invadido buena parte del norte de Grecia y, más aún que como señal de su triunfo presidiría los sacros Juegos Píticos, en Delfos. La crítica lapidaria de Demóstenes a los atenienses señala que ni siquiera de las cosas que les quedan han sacado alguna utilidad, porque lo útil, en el fondo, examinado a la luz del presente político, se vuelve un problema

no todos consideramos lo útil de una misma manera con respecto a los mismos asuntos, sino que a unos parece de este modo y a otros de otra manera.

En estas expresiones no hay un puro recurso retórico; queda formulado, aquí, el problema esencial de todo juicio y determinación política, en tanto que respuesta a un presente como aquí y ahora ya. En el fondo, esta es la aporía deinótera de la política y por consiguiente la de todo hombre que interviene en el ejercicio de la misma.

\footnotetext{
${ }^{6}$ Discurso de Demóstenes acerca de la paz. Traducción de H. García. Bizantino Nea Hellás 25, 2006:13-34.
} 
Si nos atemos a esta simple constatación, entonces podemos encontrar muchos temas e indicaciones que Aristóteles abordó no sólo en los libros éticos, sino también en los políticos, bajo la simple consideración como un referirse de la filosofía a cosas humanas y descubrir al mismo tiempo por qué el filósofo, parafraseándolo, no sólo debe ser un amante de los mitos, sino también de la historia, porque como dice el proverbio griego ólbios hóstis tês historias ésche máthesin (dichoso aquel que ha tenido conocimiento de la historia).

El análisis histórico y, por consiguiente su registro e interpretación, está en la base de sus reflexiones y en todo lo que escribió. La concepción aristotélica y propuesta política acerca de una politeía, es decir, de un régimen político óptimo, es una propuesta fundada en un concienzudo trabajo humanista en que vemos a un Aristóteles historiador, a un Aristóteles filólogo, a un Aristóteles psicólogo, en fin... al filósofo, es decir, a partir de un trabajo que se hace cargo de construir el propio método de investigación y, de hacer filosofía a la vez; en ello juega un papel fundamental la historia, su modo de abordarla y sobre la cual construye y deja conocimiento. El decurso de la historia está a la base de toda su concepción de la filosofía práctica o como él dice una vez filosofía política. En este sentido, para Aristóteles el político debe ser un conocedor de las acciones políticas que el hombre ha implementado en el percurso de la misma política. Así, en su Retórica nos encontramos con la siguiente observación:

Es útil con respecto a las legislaciones para el que investiga (theoroýnti) no sólo oír cuál régimen político conviene a partir de los que han pasado antes (ek tôn parelelythóton), sino también conocer las [constituciones] de otros; cuáles armonizan con cuáles. De modo que es evidente que con respecto a la legislación (pròs mèn tèn nomothesían) útiles son los viajes alrededor de la tierra [mundo] (hai tês gês períodoi) (de ahí, pues, es posible comprender las leyes de los pueblos) y, con respecto a las deliberaciones políticas (politikàs symboylàs), las historíai de los que escriben en relación a las acciones (hai tôn perì tàs prákseis grafónton 
historíai): todas estas cosas son trabajo de la política, pero no de la retórica ${ }^{7}$

El tiempo transcurriendo a través de los acontecimientos para verterlo en un concepto como el de la legislación. Para su captación todo es útil, no sólo la escritura, sino hasta los viajes, porque lo que interesa es tener una cosmovisión de lo que el hombre ha hecho, porque por sus acciones los hombres se hacen a sí mismos grandes (si se quiere), pero también se destruyen. Hay ahí los fundamentos de una ética y una política. En nuestra opinión, de aquí arranca la propia expresión aristotélica de filosofía política tal como la hallamos en su Política. Esta cosmovisión es posible por las historias, que en su forma plural se entienden como relatos, en especial quien se haga cargo de las deliberaciones políticas y siempre las deliberaciones políticas son en torno al presente, aunque sus consecuencias comprometan el futuro, que es de suyo un grande problema, útiles son las historíai (puro lenguaje en nuestra opinión). Decir que la historia es relato es quedarnos con uno solo de entre sus sentidos ¿Qué era historia para los griegos? Desde Tucídides, el synégrapse directo sobre el asunto ${ }^{9}$. Es esencialmente examen, indagación, interrogaciones, que en su conjunto conforman conocimiento(s); y quien escribe, no escribe sobre cualquier cosa, sino que con respecto a las acciones que emprende el hombre en su vida social, es decir, política.

En la misma Retórica ${ }^{10}$ nos encontramos con una referencia que hace a la historíe herodotea, la enmarca en lo que el filósofo llama una exposición de estilo seguido, que no tiene fin por sí mismo si no termina el asunto expuesto, añade que de este sentido de la historíe, antes, se servían todos, pero que ahora no muchos recurren a él. De hecho él y Tucídides le han dado un nuevo giro a la palabra historia. El origen de la palabra es homérico, remite a histor. Según una información, Aristóteles habría referido el plural hístoras a "hombres sabios y competentes, conocedores y expertos de las acciones y las

\footnotetext{
${ }^{7}$ Ret. $1360{ }^{\text {a }} 31-38$. Cfr., además 1365 b 23-27 donde Aristóteles recomienda que en relación al persuadir $\mathrm{y}$ al aconsejar (pròs tò dinastía peíthein kaì kalôs symboyleýein), lo mayor y más importante de todo es comprender todos los regímenes políticos (tàs politeías hapásas labeîn), así como distinguir los caracteres en cuanto a las costumbres y cosas convenientes.

${ }^{8}$ P. 3. 12.1282 b 23

${ }^{9}$ Thoykydídes Arhenaîos synégrapse tòn pólemon tôn Peloponesíôn kaì Athenaíôn hôs epolémesan pròs alléloys. I. 1, Introducción.

${ }^{10} 1409^{\text {a }} 28$
} 
pasiones humanas y que intelectualmente se distinguían de otros"11. Si la palabra historia encierra todos estos sentidos, entonces es mucho más que mero relato, a no ser que relato comprenda éstos y otros sentidos.

Aristóteles no nos entrega una definición -como suele hacerlo con su lenguaje técnico - de la palabra historia. Opera, sin embargo, desde aquel profundo sentido heleno en el que se tiene que dejar oír todo lo que compete a lo anthrópino, que desde Heródoto consiste en la narración de las vicisitudes humanas. Para Aristóteles, la historia es examen, investigación, camino a la síntesis de un conocimiento integrador, tanto de aquello que deviene de la escritura como de aquellas experiencias referidas en lo que Aristóteles llama las tà legómena, que no siempre deben entenderse como pura oralidad. El examen de estas cuestiones, las historíai, no es nada simple, según Aristóteles, como nos dice en la Ética Nicomaquea:

[...], pero los que, sin ningún hábito, recorran tales documentos [ las constituciones políticas ], no podrán juzgar acertadamente (a no ser que tengan especial don natural para ello), si bien quizá pueden adquirir de ese modo mayor comprensión de tales cuestiones $^{12}$

No tratamos de zanjar aquí la visión que Aristóteles haya tenido acerca de la historia, pero de lo que no nos cabe duda es que su filosofía política ha nacido de su información histórica y, al mismo tiempo los resultados que él ha conseguido en este campo hacen que pueda ser visto como la fuente histórica en que otros han confluido. De hecho, los elementos que aparecen en el corpus de la Política y fundamentalmente la Athenaíon Politeía, constituyen una fuente de primerísimo orden para leer y reconstruir los estadios de la institucionalidad política del mundo griego. La lectura que hace Aristóteles está orientada a descubrir el entramado de las leyes, según las constituciones, porque "en las leyes está la salvación de la ciudad", que como asociación (koinonía) está orientada para "vivir bien"13. A su propuesta política, Aristóteles la llamó simplemente politeía. Antes de referirnos a ella es preciso abordar un punto particular que dice relación con la lectura que

\footnotetext{
${ }^{11}$ Cf. Fusai, Sergio: Il Processo homérico: Dall' histor homérico all' historíe erodotea. CEDAM. Padova 2006, pág. 51 y nota 27 en particular.

${ }^{12}$ E. N. 10.9 .1181 b $10-12$

${ }^{13}$ P. 1252 b 30
} 
Aristóteles hace de la propia historia helena antigua y su vinculación con su propia propuesta de régimen político.

Hallamos en la Athenaíon Politeía la siguiente descripción sobre los caracteres que presentaba la comunidad política de los primeros tiempos. Se trata de un prolongado tiempo de conflictos entre los notables y la gente común, en el marco de un régimen político oligárquico, al que en Política califica de lían ákraton, es decir, "demasiado absoluto"14

Después de estos sucesos tuvo lugar un prolongado período de conflictos (synébē stasiásai) entre los notables y la gente común. Y es que su régimen político era oligárquico en todos los demás aspectos, pero particularmente en que los pobres, sus hijos y sus mujeres eran sometidos a servidumbre por los ricos; se les llamaba "allegados" y "sectarios" -porque labraban por esta imposición de la sexta parte las tierras de los ricos, ya que la tierra estaba en poder de unos pocos (pâsa gê di olígōn ên) - y, si no pagaban las rentas, podían ser sometidos a detención, tanto ellos como sus hijos. En cuanto a los préstamos, se hacían para todos bajo la garantía de sus personas (epì toîs sōmasin), hasta la época de Solón, que fue el primer líder popular. Por tanto, para la mayoría lo más penoso y lo más amargo del régimen político era la servidumbre, aunque también llevaban muy a mal lo demás, ya que, por así decirlo, no participaban de $n a d a^{15}$.

¿Qué es lo que concretamente hace aquí Aristóteles? En términos muy amplios, está leyendo e interpretando el pasado de Atenas. A esto podríamos llamar historia. Aristóteles lee su propia historia. No se trata de cualquier lectura, sino de una lectura política, de acontecimientos políticos y religiosos, que dibujan el estadio de una sociedad de hace casi tres siglos anteriores a él, respecto a costumbres y a leyes. Retoma un punto sensible sobre el origen de las revoluciones intestinas (las stáseis) dentro de la aún naciente sociedad

\footnotetext{
${ }^{14}$ P. 121273 b 37.

${ }^{15}$ 2. 1-3 Cf. Aristóteles, Constitución de los atenienses. Edición bilingüe de Alberto Bernabé Pajares. Abada Editores. Madrid, 2005.
} 
ateniense. Los gnōrímoi (los notables) y el plēthos (la multitud, la gente común) entraron en stásis (stasiásai) y durante mucho tiempo. La causa del problema la halla el filósofo en el ejercicio de una oligarquía extrema, dueña no sólo del poder político, sino también del principal de los bienes materiales, a saber, la posesión de la tierra, de donde derivará, a su vez, un sistema económico y social que desembocará en la implantación de la esclavitud por deuda, sistema que se proyecta durante más de siglo y medio hasta los tiempos en que emerge la figura de Solón.

El conflicto del que trata aquí, Aristóteles, es el de las staseis, es decir, el de las guerras civiles (revoluciones), que todas las ciudades del mundo griego conocieron y que Platón llamó de un modo genérico pólemos, llegando a decir que era un asunto que estaba tan íntimamente unido a todos y durante toda la vida con respecto a todas las ciudades griegas, de modo que parecía vivirse siempre bajo un estado de guerra no declarado (pólemon akérykton $)^{16}$. Por lo que sigue en el análisis de Aristóteles, se puede resumir diciendo que el problema central es el abuso por parte de los ricos - los préstamos se hacían bajo compromiso de los propios cuerpo, dicho literalmente - sobre una clase social venida a menos, los pobres. Pero el problema es más profundo aún: se trata de la desigualdad radical en el uso y distribución de la propiedad - la tierra estaba en poder de unos pocos - dice Aristóteles. A la luz de este problema el filósofo sacará varias lecciones, que encontramos desarrolladas en su Política, donde consignó importantes reflexiones en particular al tema de la propiedad y su rol, tanto en la estabilidad como en la inestabilidad de los regímenes políticos. Éste no ha sido un problema menor para la política y los políticos de todos los tiempos, como es evidente. Una de aquellas conclusiones que comenta Aristóteles en Política es que la pobreza engendra la guerra civil (stásin) y la maldad (kakoyrgían $)^{17}$, criticando, por ejemplo, la falta de cuidado en el control de la natalidad. Pero también le permitirá afirmar que la causa de la revolución tiene su origen en la opresión ejercida contra el pueblo, por la desviación de la justicia dentro del régimen aristocrático y también por el mayor abuso de las clases superiores, puesto que la propiedad está en manos de unos pocos y pueden hacer lo que se les antoja. En las democracias, la causa estaría en la asélgeia (insolencia, descaro, desvergüenza) de los demagogos.

Al revisar históricamente las constituciones, Aristóteles encuentra que han surgido diversas propuestas para enfrentar el problema de la distribución

\footnotetext{
${ }^{16}$ Véase Leyes I, 625 e y 626 a.

17 P. 2. 6. 1265 b12
} 
de la propiedad. Platón había propuesto que debía permitirse la posesión de la propiedad hasta un cierto límite. Pero el primero en plantear el problema habría sido Faleas de Calcedonia al señalar que las posesiones de los ciudadanos deben ser iguales (ísas tàs ktéseis), apoyando su propuesta en que esa igualdad debería darse tanto en el plano educacional como en el de la propiedad misma. Aristóteles a renglón seguido somete a crítica estas propuestas, señalando que aún cuando el legislador estableciera una propiedad moderada, no se ganaría nada en ello, porque es más preciso igualar las ambiciones que la propiedad, a través de una educación suficiente por medio de las leyes. La igualación en la propiedad no tiene ninguna importancia ante la ambición de los hombres, porque es insaciable, ápeiros gàr he tês epithymías phýsis ${ }^{18}$. Pero ¿cuál es entonces la propuesta de Aristóteles? Apunta a una reforma que regule que los ciudadanos superiores, los ricos "no quieran poseer más (mē boýlesthai pleonekteîn) y que las clases bajas no puedan; es decir, que sean inferiores pero sin injusticia"19

Esta propuesta de reforma, por decir lo menos, es insólita; no resiste la menor crítica, aunque acorde al propio parecer del estagirita. Quedémonos mejor con la imagen del problema en sí (el de la oysía) y por último si es posible o no encontrar una solución al problema de fondo planteado, el de la insaciable ambición humana y si la educación y la política han dado respuesta, porque aún estamos en tal aporía. Este problema reaparecerá cuando Aristóteles defina los caracteres de su politeía ideal.

Un asunto que debiera llamar la atención en ésta es a quiénes Aristóteles considera como polîtai, ciudadanos. En el libro IV (VII) nos dice, en primer lugar, que la ciudad debe poseer un elemento armado y uno deliberativo $^{20}$ y que en el fondo corresponderían al poder ciudadano. Pero éstos en tanto que tales no pueden llevar vida de obreros (artesanos), mercaderes, ni labradores ${ }^{21}$, porque sería contrario a la virtud, y argumenta diciendo que para que surja la virtud como actividad política es indispensable el ocio; por consiguiente, los pobres están imposibilitados de acceder a tal virtud política. Los ciudadanos deben tener la propiedad ${ }^{22}$ como una necesidad, porque ellos son los eyporoi, es decir, los ricos, deben disfrutar del ocio. En definitiva ellos son la clase productora de virtud. Ninguna clase -dice

\footnotetext{
${ }^{18}$ P. 2. 7.1267 b 3-4

${ }^{19}$ P. 2. 7- 1267 b 7-9

20 P. $1329^{\text {a }} 2$ ss.

${ }^{21}$ P. 1328 b 34 ss.

${ }^{22}$ P. $1329^{\text {a }} 17$ ss.
} 
Aristóteles- que no sea productora de virtud es clase ciudadana ${ }^{23}$. Más adelante considera nuevamente el tema de la distribución de la tierra. Para él debe ser privada con una cierta distribución sólo en la clase ciudadana, quienes contarán con una porción de ella tanto en la ciudad como en las fronteras; ello aseguraría, naturalmente, el interés por la defensa del territorio. Los únicos indicios de un sentido nacional del uso de la tierra los hallamos cuando Aristóteles deja una porción destinada a sufragar el costo del culto público y de las comidas en común, que considera son medios necesarios para promover la unidad del Estado. Pero también habría que decir que en ese libro IV (VII) el culto de los dioses Aristóteles lo reserva como función exclusiva sólo de los ciudadanos, es decir, de los eyporoi, que ya se hayan retirado por la edad ${ }^{24}$. Como vemos, en la óptica de la ciudad mejor de Aristóteles sigue primando una distribución de la sociedad, basada fundamentalmente entre ricos (eyporoi) y pobres (áporoi). De aquí que pueda decirse que este modelo es el más puro modelo de la originaria política aristocrática, desarrollada en el mundo griego. Sin embargo, a este modelo añadirá un elemento novedoso.

En el libro VI (IV) agrega un tercer elemento que llama mesoi, es decir, los intermedios o clase media, en nuestro lenguaje $\mathrm{e}^{25}$. En ninguna parte de la Política, Aristóteles nos ha dicho qué y quiénes representan la clase media, pero al parecer podría pensarse en aquellos que ocupan una posición intermedia con respecto a las posesiones. No basta la fusión de elementos provenientes de la oligarquía ni de la democracia para la conformación del régimen mixto que propone Aristóteles, sino que se requiere de este tercer componente que es la clase media, que demarca la equidistancia entre los dos extremos $^{26}$. Aristóteles aquí ha hecho prevalecer aquel principio de la virtud, entendida como medio entre los extremos, que plantea en la Ética Nicomaquea, y que la recuerda expresamente aquí ${ }^{27}$, la vida feliz es la vida del justo medio. Por ello ahora Aristóteles señala que en el extremo de los muy ricos prima la soberbia e incluso la maldad; por su parte, en el de los pobre se dan los malhechores y los capaces de pequeñas maldades. Aristóteles argumenta que donde predomina el primer elemento se crea una sociedad de esclavos y amos y esto se aparta lejos de la amistad, "porque la comunidad implica la amistad" 28 . A nuestro entender es este un concepto clave en el que

\footnotetext{
${ }^{23}$ P. $1329^{\text {a }} 20$

${ }^{24}$ P. $1329^{\text {a }} 32-34$

${ }^{25}$ P. 1295 b $1-3$

${ }^{26}$ P. 1295 b $33-34$

${ }^{27}$ P. $1295^{\text {a }} 35-40$

${ }^{28}$ P. 1295 b $21-24$
} 
ha decantado la profunda y prolongada reflexión de Aristóteles sobre el tema de la amistad, tal como la encontramos en los libros de ética. De momento limitemos a destacar el principio fundamental de la amistad, a saber, que es lo más esencial y hermoso para la vida: "Sin amigos -dice el filósofo- nadie querría vivir, aun cuando poseyera todas las demás cosas"29. Tanto en la riqueza como en la pobreza requerimos de ella. Si en nuestra vida individual es fundamental, como mayor razón lo será para la comunidad política:

Parece además que la amistad -prosigue el estagirita-mantiene unidas a las ciudades, y que los legisladores consagran más esfuerzos a ella que a la justicia: en efecto, la concordia parece ser algo semejante a la amistad, y es a ella a lo que más aspiran, mientras que lo que con más empeño procuran expulsar es la stásis, que es odiosidad. ${ }^{30}$

En una utopía filantrópica el filósofo llega a pensar que con la amistad sin más no habría necesidad de la justicia, pero no a la inversa, porque aún siendo justos, requerimos de la amistad ${ }^{31}$. Es indispensable, entonces, que la ciudad se construya sobre la mayor cantidad de elementos iguales, dan la concordia. Esos elementos iguales son el aporte de la clase media, porque es la que más fácilmente obedece a la razón ${ }^{32}$. No ambiciona en exceso los cargos públicos, pero tampoco los rehuye. Los gobiernos, fundados en la clase media, son los más estables y mientras mayor sea su número habrá mejor comunidad política, mejor gobierno, porque "produce el equilibrio e impide los excesos contrarios"33. Aquí Aristóteles propone que los ciudadanos tengan una hacienda medida y suficiente, porque de lo que se trata es de no permitir el engrandecimiento desproporcionado de ninguno de los politai ${ }^{34} \mathrm{y}$ hacia este objetivo debe apuntar la legislación. Aristóteles piensa que un remedio en tal dirección es recomendar las empresas y distribuir las funciones administrativas a elementos diversos, inclusive opuestos, es decir, se trata de intentar la mayor combinación de pobres y ricos y esos significa aumentar la

\footnotetext{
${ }^{29}$ E. N. $8.11155^{\text {a }} 5 \mathrm{ss}$.

${ }^{30}$ E. N. 8. $1.1155^{\text {a }} 22-26$, cfr. E. E. VIII $1155^{\text {a }} 22-24$.

${ }^{31}$ E. N. $1155^{\text {a }} 26-28$

${ }^{32}$ P. 1295 b $5-6$

${ }^{33}$ P. 1295 b $33-34$

${ }^{34}$ P. 1308 b $10-12$
} 
clase media, porque "así se deshacen las stáseis (las guerras civiles) que tienen por causa la desigualdad" 35 .

Aristóteles, en su alabanza a una politeía mixta, constituida sobre la clase media, hace pesar la tradición histórica de la experiencia política del mundo griego, en su tiempo, de una manera profundamente sentida, porque la guerra del Peloponeso había exterminado el incipiente progreso social y con ello el desarrollo de la propia clase media, Aristóteles concluirá su fundamentación diciendo:

Que el régimen intermedio es el mejor, es evidente, puesto que es el único libre de sediciones (astasíastos). En efecto, donde la clase media es numerosa es donde menos sediciones (stáseis) y disensiones civiles (diastáseis) se producen, y las grandes ciudades están más libres de sediciones por la misma razón, porque la clase media es numerosa (hóti poly tò mesón) [...] Las democracias son también más firmes y duraderas que las oligarquías por las clases medias ${ }^{36}$.

Una filosofía que se proponga hablar realmente de las cosas humanas, en particular cuando se trata del modo cómo podemos lograr el vivir bien en ciudad, es necesario que a lo menos se haga cargo de replantearse las mismas cuestiones de las que nos hemos hecho cargo en esta comunicación, a la luz orientadora del pensamiento del estagirita, porque es el modo de suscitar nuevas propuestas ante nuevos y más agudos problemas, por más que frente a lo útil tengamos diferentes modos de responder.

Sirvan estas notas para hacer sonar que los temas de la política griega nos siguen urgiendo en nuestros días y que las cuestiones planteadas por Aristóteles nos ponen una cota de permanente revisión. Con la pérdida de una literatura universal en la formación humanista y, en el campo de la historia, la pérdida de la historia universal, griega por qué no decirlo, hemos perdido también una formación política, que es la que dan las literaturas antiguas, en particular la helena ${ }^{37}$

\footnotetext{
${ }^{35}$ P. 1308 b $24-31$

${ }^{36}$ P. $1296^{\text {a }} 7-15$

37 Asunto que ya había advertido nuestra Gabriela Mistral ante la cancelación del latín en los programas de educación, aquí, en Chile, quien consideraba una pérdida no
} 


\section{BIBLIOGRAFÍA}

\section{TEXTOS-FUENTE}

ARISTÓTELES, La Constitución de Atenas. Edición bilingüe por Antonio Tovar. Instituto de Estudios Políticos. Madrid, 1948.

- $\quad$ Constitución de los Atenienses. Edición bilingüe de Alberto Bernabé. ABADA Editores. Madrid, 2005.

- $\quad$ Ética Nicomaquea. Edición bilingüe y traducción por María Araujo y Julián Marías. Instituto de Estudios Políticos. Madrid, 1970.

- $\quad$ Política. Edición bilinguie y traducción por Julián Marías y María Araujo. Centro de Estudios Constitucionales. Madrid, 1983.

- $\quad$ The Politics of Aristotle. With an Introduction, two Prefatory Essays and Notes Critical and Explanatory. Por W. L. Newman. At the Clarendon Press. Oxford,1950.

- $\quad$ Politique. Texte établi et traduit par Jean Aubonnet. «Les Belles Lettres ». Paris, 1968.

- Retórica. Edición del texto con aparato crítico, traducción, prólogo y notas por Antonio Tovar. Instituto de Estudios Políticos. Madrid, 1971.

sólo literaria, sino también política, "pues leyendo a sus clásicos, se aprende democracia”. Artes y Letras, El Mercurio, domingo 30 / XII / 2007, pág. 8. 